P103 (continued)

$(\mathrm{p}<0.05)$ and for fruits by 0.39 cups $(\mathrm{p}<0.01)$ at 1 week follow up. Participants also increased utilization of weekly meal planning techniques from $14.8 \%$ to $50 \%(\mathrm{p}<0.01$ ), increased physical activity for more than 30 minutes during 7 days a week from $16.7 \%$ to $36 \%(\mathrm{p}<0.01)$ and increased consumption of water with all meals from $39 \%$ to $70.6 \%$ ( $<<0.01$ ) when comparing baseline to 1 month follow up. Conclusions and Implications: The implementation of a goal-setting nutrition education program improved the level of knowledge, self-efficacy and behavior among low-income SNAP-eligible adults.

Funding: Supplemental Nutrition Assistance Program Education

\section{P104 Factors Associated With Cue-Elicited Food Craving Among Elementary School Children}

GauriKarnik,DTR, gkarnik@mail.csuchico.edu, California State University-Chico, Tehama 124, Chico, CA 95929;

K. Goto, PhD; B. Seipel, PhD; S. Pierson, BS;

J. Giampaoli, PhD; K. Buffardi, PhD

Objective: To examine factors associated with cue-elicited food craving among low-income elementary school children.

Design, Setting, and Participants: A cross-sectional study was conducted with 247 third-to-fifth graders primarily consisting of Hispanic and non-Hispanic white students. The study was held in their classrooms where each participant had access to a laptop computer.

Outcome Measures and Analysis: Cue-elicited craving of high-calorie, palatable food was measured using a software program that presented 12 food images. Participants viewed each food image and rated on a five-point scale how much they wanted to eat the food they had just seen. Next, participants selected the image of the food they craved most out of 18 culturally appropriate images. Participants' food intake and demographic characteristics were also assessed. Independent t-tests, one-way ANOVA, Pearson's correlation and chi-square were used for statistical analysis.

Results: Male students reported significantly higher cueelicited food craving than females. Fifth graders reported significantly less food craving compared to their counterparts. Ethnicity was not associated with food craving. Food craving was significantly correlated with sugar-sweetened beverage and sweet intake and inversely correlated with vegetable intake. Additionally, $40.5 \%$ of the participants reported Takis (tortilla snacks) as the most craved food and $11.7 \%$ chose pizza. Grade was significantly associated with types of craved food.

Conclusions and Implications: Cue-elicited food craving appears to be a factor associated with palatable food intake among children. More research is warranted to understand socio-cultural and environmental factors affecting food craving and its influence on overeating and obesity.

Funding: USDA NIFA AFRI
P105 Identification of Preferences for Bread Shapes in Young Children

Lauren Keeney, MS Candidate, laurenkeeney@vandals. uidaho.edu, University of Idaho, 875 Perimeter Drive, Moscow, ID 83844; $S$ Ramsay, $P h D, R D, L D N$;

L. L. Tsao, PhD; S. Planck, PhD

Objective: Cognitive development theory suggests that a single attribute can influence preferences in young children. The purpose of this study was to determine whether preferences and intake of a palatable food is different when presented in altered shapes.

Design, Setting, Participants, and Intervention: Children 3-5 years of age $(n=21)$ were recruited from a University Child Development Laboratory and participated in a hedonic taste preference activity. Each child was offered four 50-gram bread samples prepared from the same recipe. Different shapes were created: bread stick, knotted roll, cloverleaf roll, and butterflake roll, and children were allowed to eat as much as they wanted.

Outcome Measures and Analysis: Bread shapes were weighed post activity as a proxy for intake and children's reported preferences were recorded. Spearman correlation determined whether children's preference for each bread was related and an ANOVA determined the difference in children's consumption.

Results: Children's preferences were not consistent across shapes, $86 \%$ of children $(n=18)$ reported the breadstick as "yummy", while $48 \%$ of children $(n=10)$ reported the clover roll as just okay/yucky. Children's liking for the bread stick and knotted roll were positively correlated (rs $[22]=.61, \mathrm{p}<.01)$, while the bread stick was negatively correlated with the clover $(\mathrm{rs}[22]=.8, \mathrm{p}, 0.01)$ and butterflake ( $\mathrm{rs}[22]=.75, \mathrm{p}<.01)$ rolls. children's consumption was not significantly different, but mean intakes varied: $4.1 \mathrm{~g}$ breadstick, $3.1 \mathrm{~g}$ knotted roll, $3.5 \mathrm{~g}$ cloverleaf, and $3.2 \mathrm{~g}$ butterflake.

Conclusions and Implications: In a young child, a single attribute (shapes) influences preference and may affect intake. Caregivers who feed young children should recognize how a single attribute (i.e. how food is presented) can influence the foods liked and consumed by children.

Funding: Idaho Wheat Commission

\section{P106 Parenting Practices That Support Young Children's Preference for Varied Healthful Foods}

Jane Lanigan, PhD, jlanigan@wsu.edu, Washington State University, 14204 Northeast Salmon Creek Avenue, Vancouver, WA 98686; V. Jarvensivu, MS; R. Bailey, PhD; S. Ramsay, $P h D, R D N, L D$, University of Idaho

Objective: Eating a variety of healthful foods support children's health and growth. This study examined how parent feeding practices influence young children's preference for a variety of healthful foods.

Design, Setting, and Participants: Children ages 3-5 $(\mathrm{N}=46)$ attending a university child development program 